\title{
The association of loneliness after sudden bereavement with risk of suicide attempt: a nationwide survey of bereaved adults
}

\author{
Alexandra L. Pitman ${ }^{1,2}$ - Michael B. King ${ }^{1} \cdot$ Louise Marston $^{3} \cdot$ David P. J. Osborn ${ }^{1,2}$
}

Received: 10 January 2019 / Accepted: 8 July 2020 / Published online: 18 July 2020

(c) The Author(s) 2020

\begin{abstract}
Purpose We aimed to test the hypothesis that among people who experience sudden bereavement, loneliness is associated with post-bereavement suicide attempt and post-bereavement suicidal ideation, even when adjusting for network size.

Methods We analysed cross-sectional data collected in the 2010 UCL Bereavement Study, to identify 3193 respondents who had experienced sudden bereavement. We used multivariable logistic regression to test for an association between loneliness (using a newly-developed eight-item loneliness measure) and post-bereavement suicide attempt and suicidal ideation, adjusting for socio-demographic factors, pre-bereavement depression and self-harm, and network size.

Results Among bereaved adults, loneliness was significantly associated with probability of post-bereavement suicide attempt (AOR 1.19; 95\% CI 1.14-1.25) and of post-bereavement suicidal ideation (AOR 1.24; 95\% CI 1.20-1.28), with estimates unchanged by adding perceived stigma of the bereavement to adjusted models. There was no association between suicide bereavement and loneliness (adjusted coefficient $0.22 ; 95 \% \mathrm{CI}-0.12$ to $0.45 ; p=0.063$ ). The association of loneliness and suicide attempt risk was similar whether participants were bereaved by suicide or not.

Conclusions People who report feeling lonely after sudden bereavement are more likely to make a suicide attempt after their loss, even when taking into account their network size and the perceived stigma of the sudden bereavement. There is no evidence that the effects of loneliness on suicidality are specific to suicide bereavement. This work identifies loneliness as a potential target for suicide prevention interventions among bereaved people. It also fuels interest in longitudinal research investigating loneliness as a putative mediator of suicide risk.
\end{abstract}

Keywords Loneliness $\cdot$ Social isolation $\cdot$ Suicide attempt $\cdot$ Suicidal ideation $\cdot$ Stigma $\cdot$ Bereavement

This article is part of the focused issue 'Loneliness: contemporary insights on causes, correlates, and consequences'.

Electronic supplementary material The online version of this article (https://doi.org/10.1007/s00127-020-01921-w) contains supplementary material, which is available to authorized users.

Alexandra L. Pitman

a.pitman@ucl.ac.uk

1 UCL Division of Psychiatry, University College London, Maple House, 149 Tottenham Court Road, London W1T 7NF, UK

2 Camden and Islington NHS Foundation Trust, St Pancras Hospital, 4 Saint Pancras Way, London NW1 OPE, UK

3 UCL Research Department of Primary Care and Population Health, University College London, Gower St, London WC1E 6BT, UK

\section{Introduction}

The World Health Organisation (WHO) reports that 800,000 people die by suicide annually worldwide, and that for each of those deaths approximately 20 other people attempt suicide [1]. Amongst long-established risk factors such as mental illness and alcohol misuse, the WHO also hypothesises that a sense of isolation may be a risk factor for suicidality [1]. Whilst social isolation is defined objectively as a lack of social contacts, the concept of loneliness has been defined as the unpleasant experience that occurs when a person's network of social relationships is deficient either quantitatively or qualitatively [2]; the subjective, unwelcome feeling of a lack or loss of companionship [2]; and as an inner, subjective sense of not being sufficiently socially connected [3]. Loneliness and social isolation are both distinct from the concept of social support; the emotional, tangible (practical), informational, and companionship support arising 
from either formal (professional) or informal (friends and relatives) sources [4]. Both social isolation and loneliness have recently become the focus of health and social policy research, and the adverse physical and mental health effects of loneliness are becoming increasingly well recognised [5], including the association between loneliness or social isolation and suicidality [6]. This is a particular concern amongst young people, in whom loneliness is prevalent and also stigmatising [5]. The hypothesised links between loneliness and suicidality are that lonely individuals feel dissatisfied with life [7], and that their perceptions of limited social connections contribute to a desire to die [8]. Other mechanisms suggested include the contribution of loneliness to chronic stress, the link with cognitive factors such as low self-esteem or an external locus of control, and the mediating role of depression [6]. Theoretical models of suicidality consider the role of thwarted belongingness; a construct describing what arises when the fundamental need to form and maintain strong, stable interpersonal relationships is unmet, resulting in feelings of disconnection [8, 9]. The Interpersonal Theory of Suicide considers thwarted belongingness as comprising (a) loneliness and (b) the absence of reciprocal caring relationships [8], and confirmatory factor analysis shows convergent associations of thwarted belongingness with loneliness [10]. The Integrated Motivational-Volitional (IMV) model of suicide proposes that factors such as thwarted belongingness and perceived burdensomeness (the view that one's existence is a burden on friends, family and/or society) govern the transition from suicidal ideation to suicide attempt [11]. Studies conducted to date have found loneliness to be associated with suicidal behaviour in Canadian [12] and English [6] general population samples, even when adjusting for common mental disorder [6], and in specific populations limited to war veterans [13, 14], adolescents [15], and sexual minority groups [16]. However, no studies have investigated this in a bereaved population.

The loneliness arising from sudden bereavement is a topic worthy of further investigation because sudden bereavement is itself a risk factor for suicide [17], particularly where the death was due to suicide $[18,19]$. Although the mediators of suicide risk after sudden bereavement remain unidentified, factors such as loneliness, social isolation, and poor social support are implicated [20]. Loneliness after sudden loss may arise from the abrupt loss of a confiding relationship or companionship. The taboo around sudden or violent losses can also engender social isolation due to others' awkwardness and avoidance [21]. The perceived stigma of sudden bereavement, defined as the subjective awareness of others' negative attitudes towards the loss [22], is likely to be a major contributor to loneliness after loss. This stigma arises not only from others' avoidance but also from a perception of them gossiping about the death or casting blame [23]. Although stigma is perceived after all sudden deaths, most commonly due to embarrassment or a fear of appearing socially incompetent, it is particularly pronounced after deaths by suicide and other unnatural causes [24, 25]. This is likely to be due to the long history of religious and cultural sanctions against suicide, stigmatising the act and those mourning it [26], and others' views about irresponsibility in the case of accidental death [21]. The perceived stigma of sudden bereavement is associated with risk of suicidal thoughts and suicide attempts [22], and there is also evidence that perceived stigma contributes to suicide attempt risk after suicide bereavement [25].

In addition to suicidality, unexpected bereavement is also associated with a range of incident psychiatric disorders [27], and it is possible that these might lead to or compound loneliness. Not all individuals will be adversely affected, and grief is a non-pathological process. However, risk of poor outcomes is likely to be governed by factors such as personal vulnerability [28], the quality of attachment to the person who died [29], and the degree to which social support buffers the negative impact of a traumatic life event [30]. Further work is needed to describe the relationships between loneliness, social isolation and suicidality after a sudden loss to further our understanding of the roles that social isolation and loneliness might play in the pathway to suicidality. No previous studies have measured the association between loneliness after the sudden loss of a close friend or relative and suicide-related outcomes. We aimed to investigate whether greater loneliness was associated with a higher probability of suicide attempt and suicidal ideation after sudden bereavement in a population-based sample of adults in the United Kingdom. We also aimed to test the hypothesis that associations differ by mode of bereavement (suicide bereavement versus non-suicide bereavement), such that the magnitude of the association would be greater in the suicide-bereaved, and that the main association might partly be accounted for by the perceived stigma of the bereavement.

\section{Methods and materials}

\section{Study design}

We analysed cross-sectional data collected in the UCL Bereavement Study: a 2010 survey of all staff and students aged 18-40 at 37 UK higher educational institutions (HEIs) who had experienced the sudden bereavement of a close friend or relative since reaching the age of 10 . Full details of sampling for this closed online survey have been described elsewhere [22], including the survey instrument [25]. The focus was on young adults given policy concerns about their risk of suicide [31] and their tendency not to engage with services when in distress [32-34]. Sampling via global email lists avoided the biases associated with recruiting a 
help-seeking sample, and was felt to be the most efficient, comprehensive and pragmatic means of recruiting a hardto-reach population of young adults [35].

Of 5085 respondents to the survey, we included those who: consented to participate; completed the loneliness item of the Social Functioning Questionnaire (SFQ) and all seven items within a scale measuring perceptions of social support received from family and friends; and specified their mode of bereavement $(n=3193)$. This sample included respondents who had experienced sudden bereavement due to sudden natural causes $(n=1952)$, sudden unnatural causes ( $n=666)$, or suicide $(n=575)$.

\section{Ethical approval}

Ethical approval for analysing data from the UCL Bereavement Study was obtained from the UCL Research Ethics Committee in 2010 (reference number: 1975/002).

\section{Measures}

\section{Exposure: loneliness}

Our exposure was loneliness using eight items chosen to represent the construct of loneliness, as used in the sevenyearly Adult Psychiatric Morbidity Surveys (APMS), a regular representative population survey in England [36]. One item was taken from the SFQ [37]; an eight-item self-report scale used to measure current social function. Seven items were taken from a scale measuring perceptions of social support received from family and friends, used in the 1987 Health and Lifestyle survey in England [38]. These capture respondents' sense of whether relatives and friends can: be relied upon; make them feel loved, valued and accepted; and support and encourage them. The items therefore convey the degree to which one's relationships create a perception of social belonging and acceptance as well as of emotional connectedness, and companionship. By reverse scoring them in this loneliness measure, higher scores captured the perception of lacking belongingness, acceptance, connectedness and companionship in one's social relationships.

The development of this continuous measure, scored from 0 to 17 , is described in "Appendix".

\section{Outcome: suicide attempt}

Our outcomes were: self-reported suicide attempt ("Have you ever made an attempt to take your life, by taking an overdose of tablets or in some other way?") [39] and selfreported suicidal ideation ("Have you ever thought of taking your life, even though you would not actually do it?") [40] post-bereavement. These standardised measures were again taken from the APMS [36], qualified by whether each was before or after the sudden bereavement, or both, to derive an incident measure.

\section{Covariates}

We also measured the following covariates:

- socio-economic status, using the UK Office for National Statistics Standard Occupational Classification [41];

- depression, using the Composite International Diagnostic Interview (CIDI) screen for lifetime depression [42], qualified by whether the onset of these core symptoms of low mood and anhedonia occurred before or after the sudden bereavement, to derive an incident measure

- possible personality disorder, using the 8-item self-report Standardised Assessment of Personality-Abbreviated Scale (SAPAS), a standardised instrument with demonstrated reliability and validity in screening for likely personality disorder in psychiatric out-patient samples $[43,44]$

- perceived stigma of the sudden loss, measured using the stigmatisation sub-scale of the validated Grief Experience Questionnaire (GEQ) [26]

- network size, using a continuous measure of primary group size derived from the Interview Measure for Social Relationships (IMSR); a standardised instrument with demonstrated reliability [45], as used in the APMS [36] with waves of population norms [46]. This captures the numbers of friends and relatives (aged 16 years and over) respondents felt close to, sub-categorised into numbers of close relatives, extended family members, and friends.

We selected nine potential confounding variables on the basis of existing literature and clinical judgement: age, gender, socio-economic status, pre-bereavement depression, pre-bereavement (suicidal and non-suicidal) self-harm; and primary group size.

Missing data for model covariates and outcomes varied from $<1 \%$ (for age, gender, group size, pre-bereavement depression, pre-bereavement self-harm, pre-bereavement suicide attempt, and pre-bereavement suicidal thoughts) to $3 \%$ (for socio-economic status).

\section{Statistical analysis}

We described median values (and interquartile ranges) or means (and standard deviations) for our exposure variable and each descriptive variable, and reported $p$ values for tests of univariate associations with each using linear regression. Our threshold for significance was set at $p<0.05$.

We investigated the relationship between loneliness and outcomes using multilevel regression models with HEI as random effect, to take into account the clustering effect at 
the HEI level. We used multivariable logistic regression to investigate the relationship between loneliness (continuous measure) and two binary outcomes (post-bereavement suicide attempt, post-bereavement suicidal ideation), adjusting for socio-demographic factors, pre-bereavement psychopathology, and primary group size, as described above.

To test whether the effect of loneliness on outcomes varied by mode of bereavement (suicide versus non-suicide loss), we added this binary variable as an interaction term to adjusted models, using a less stringent $p$ value threshold $(p<0.1)$ to reflect the limited statistical power of interaction tests. We also used multivariable logistic regression to investigate whether suicide bereavement is associated with post-bereavement loneliness, adjusting for the same potential confounders as in the list above.

Finally, we tested whether adding the perceived stigma of bereavement to our main adjusted models attenuated the association between loneliness and suicide-related outcomes, to provide evidence supporting a partially mediating effect. To set this in context, we also described the association between loneliness and perceived stigma.

Models were fitted using complete case analysis. We ran a priori defined sensitivity analyses to assess the robustness of our main findings when taking into account biases introduced by $3 \%$ missing data on socio-economic status, using best-case and worst-case scenarios to impute missing values.

All analyses were conducted using Stata version 13 (StataCorp. 2013. Stata Statistical Software: Release 13. College Station, TX: StataCorp LP).

\section{Results}

\section{Participant characteristics}

The majority of our sample of 3193 adults were female (81\%), of white ethnicity (90\%), bereaved by sudden natural causes (61\%), and reporting the death of a relative (71\%) (Table 1). The sample was relatively evenly split between participants aged 18-21 years (41\%) and those aged 22-40 years (59\%). Mean age of respondents was $25 \cdot 1$ years (standard deviation [SD] 6.3, whilst mean age at bereavement was 20.1 years (SD 6.04; median 19; interquartile range [IQR] 16-23). Mean time elapsed since bereavement was 5 years (SD 5.3; range 1 day-30 years). The age of the deceased varied from 0 (for miscarriage or stillbirth) to 100 years, with a median age of 47 years (IQR 23-63). In the total sample mean loneliness scores were 2.49 (SD 2.70; median 1; IQR 1-3). Median network size in this population was 11 (IQR 7-16; mean 12.9; SD 9.1). The proportion scoring positive for possible personality disorder was $37 \%$, ranging from $22 \%$ reporting "difficulty making and keeping friends" and $22 \%$ describing themselves as a "loner", to $63 \%$ reporting being "a perfectionist" and $72 \%$ as "a worrier".

Univariate associations of loneliness with socio-demographic and clinical characteristics, and measures of social connectedness, are shown in Table 1. Loneliness scores were significantly higher in men, those who defined themselves as single or non-white, those classified as of lower socio-economic status, those reporting depression or self-harm prior to bereavement, those reporting post-bereavement suicidal thoughts or attempts, those screening positive for possible personality disorder, and those with greater perceived stigma scores.

\section{Association between loneliness scores and outcomes}

In an adjusted analysis (Table 2), higher loneliness scores were associated with a significantly higher probability of post-bereavement suicide attempts (AOR 1.19; 95\% CI $1.14-1.25$ ) and of post-bereavement suicidal ideation (AOR $1.24 ; 95 \%$ CI 1.20-1.28). Stepped adjustments are reported in Supplementary Table 3.

There was no evidence to support an interaction of mode of bereavement with suicide attempt $(p=0.7211)$ or suicidal ideation $(p=0.6343)$. Associations with suicide attempt were similar whether someone had been bereaved by suicide (AOR 1.21 ; 95\% CI $1.10-1.33 ; p=0.005$ ) or by nonsuicide causes (AOR 1.19; 95\% CI 1.12-1.25; $p<0.001$ ). Similarly, associations with suicidal ideation were similar whether someone had been bereaved by suicide (AOR 1.26; 95\% CI 1.16-1.37; $p<0.001$ ) or non-suicide causes (AOR 1.23 ; 95\% CI $1.19-1.28 ; p<0.001)$. There was no significant association between suicide bereavement and loneliness (adjusted coefficient $0.22 ; p=0.063 ; 95 \% \mathrm{CI}-0.12$ to 0.45 ), when adjusted for the same nine covariates included in the main model.

There was a significant association between loneliness and perceived stigma of bereavement, in an unadjusted model [unadjusted coefficient 0.574 (95\% CI 0.525-0.623; $p \leq 0.001)$ and when adjusting for the same nine covariates (adjusted coefficient 0.532 (95\% CI $0.480-0.583$; $p \leq 0.001)]$.

Adding scores for perceived stigma of sudden bereavement to final models (Table 2) slightly attenuated the association between loneliness and suicide attempt (AOR 1.13; 95\% CI 1.08-1.19), and between loneliness and suicidal ideation (AOR 1.18; 95\% CI 1.14-1.23), with both associations remaining significant.

We added a post hoc test for an interaction with stigma (dichotomised at the median into high versus low scores) and found no evidence for an interaction with suicide attempt $(p=0.6343)$ or suicidal ideation $(p=0.2053)$. 
Table 1 Univariate associations of loneliness scores (continuous variable) with participant characteristics $(n=3193)$
Proportion of over- Loneliness score $p$ value ${ }^{\mathrm{a}}$ all sample $n(\%)$ median (IQR)

\begin{tabular}{|c|c|c|c|}
\hline \multicolumn{4}{|l|}{ Socio-demographic characteristics } \\
\hline \multicolumn{4}{|l|}{ Gender } \\
\hline Male & $592(19)$ & $2(1,4)$ & \multirow{3}{*}{$<0 \cdot 001$} \\
\hline Female & $2600(81)$ & $1(1,3)$ & \\
\hline Missing & $1(<1)$ & & \\
\hline \multicolumn{4}{|l|}{ Age of participant (years) } \\
\hline Aged 18-21 & $1276(40)$ & $1(1,3)$ & \multirow[t]{2}{*}{$0 \cdot 065$} \\
\hline Aged 22-40 & $1917(60)$ & $1(1,3)$ & \\
\hline \multicolumn{4}{|l|}{ Relationship status } \\
\hline Single & $2238(70)$ & $1.5(1,4)$ & \multirow[t]{3}{*}{$<0 \cdot 001$} \\
\hline Within a relationship & $950(30)$ & $1(1,3)$ & \\
\hline Missing & $5(<1)$ & & \\
\hline \multicolumn{4}{|l|}{ Self-defined ethnicity } \\
\hline White & $2875(90)$ & $1(1,3)$ & \multirow[t]{3}{*}{$<0 \cdot 001$} \\
\hline Non-white & $315(3)$ & $2(1,4)$ & \\
\hline Missing & $3(<1)$ & & \\
\hline \multicolumn{4}{|l|}{ Socio-economic status ${ }^{\mathrm{b}}$} \\
\hline Social classes 1.1 and 1.2 & $1972(62)$ & $1(1,3)$ & \multirow[t]{3}{*}{$0 \cdot 179$} \\
\hline Social classes 3-7 and 9 & $1126(35)$ & $2(1,4)$ & \\
\hline Missing & $95(3)$ & & \\
\hline \multicolumn{4}{|l|}{ Educational status } \\
\hline Attained maximum A level equivalent & $1389(44)$ & $1(1,3)$ & \multirow[t]{3}{*}{0.072} \\
\hline Attained degree level or above & $1797(56)$ & $1(1,3)$ & \\
\hline Missing & $7(<1)$ & & \\
\hline \multicolumn{4}{|l|}{ Clinical characteristics } \\
\hline \multicolumn{4}{|l|}{ Post-bereavement suicidal thoughts } \\
\hline Yes & $1512(47)$ & $2(1,5)$ & \multirow[t]{3}{*}{$<0.001$} \\
\hline No & $1662(52)$ & $1(1,2)$ & \\
\hline Missing & $19(<1)$ & & \\
\hline \multicolumn{4}{|l|}{ Post-bereavement suicide attempts } \\
\hline Yes & $209(7)$ & $3(1,7)$ & \multirow[t]{3}{*}{$<0 \cdot 001$} \\
\hline No & $2958(93)$ & $1(1,3)$ & \\
\hline Missing & $26(<1)$ & & \\
\hline \multicolumn{4}{|l|}{ Pre-bereavement depression ${ }^{\mathrm{c}}$} \\
\hline Yes & $629(20)$ & $1(1,5)$ & \multirow[t]{3}{*}{$<0 \cdot 001$} \\
\hline No & $2559(80)$ & $1(1,3)$ & \\
\hline Missing & $5(<1)$ & & \\
\hline \multicolumn{4}{|c|}{ Pre-bereavement (suicidal and non-suicidal) self-harm } \\
\hline Yes & $714(22)$ & $2(1,4)$ & \multirow[t]{3}{*}{$<0 \cdot 001$} \\
\hline No & $2448(77)$ & $1(1,3)$ & \\
\hline Missing & $31(1)$ & & \\
\hline Personality disorder screen positive ${ }^{\mathrm{d}}$ & & & \\
\hline Yes & $1182(37)$ & $2(1,5)$ & $<0 \cdot 001$ \\
\hline No & $2011(63)$ & $1(1,2)$ & \\
\hline Missing & $0(0)$ & & \\
\hline Characteristics of the bereavement & & & \\
\hline Kinship to the deceased & & & \\
\hline Blood-related & $2276(71)$ & $1(1,3)$ & 0.530 \\
\hline Non blood-related & $906(28)$ & $1(1,3)$ & \\
\hline Missing & $11(<1)$ & & \\
\hline
\end{tabular}


Table 1 (continued)

\begin{tabular}{llll}
\hline & $\begin{array}{l}\text { Proportion of over- } \\
\text { all sample } n(\%)\end{array}$ & $\begin{array}{l}\text { Loneliness score } \\
\text { median (IQR) }\end{array}$ & $p$ value $^{\mathrm{a}}$ \\
\hline $\begin{array}{l}\text { Years since bereavement } \\
\text { Less than 2 years }\end{array}$ & $982(31)$ & $1(1,3)$ & 0.390 \\
$\begin{array}{l}\text { Over 2 years } \\
\text { Missing }\end{array}$ & $2211(69)$ & $1(1,3)$ & \\
$\begin{array}{l}\text { Perceived stigma of the bereavement } \\
\text { the median) }\end{array}$ & $0(0)$ & & $<\mathbf{0 \cdot 0 0 1}$ \\
High & & & \\
Low & $1525(48)$ & $2(1,5)$ & $1(1,2)$ \\
\hline
\end{tabular}

${ }^{\mathrm{a}} p$ values for univariate associations of characteristics with loneliness scores

${ }^{\mathrm{b}}$ Socio-economic status using the 5 categories from UK Office for National Statistics

${ }^{\mathrm{c}}$ Measured using CIDI screen for depression [42]

${ }^{\mathrm{d}}$ Measured using SAPAS screen for possible personality disorder [44]

${ }^{\mathrm{e}}$ Measured using stigmatisation sub-scale of the Grief Experience Questionnaire [26]

Table 2 Estimates of the associations between loneliness scores and outcomes in bereaved participants $(n=3193)$

\begin{tabular}{|c|c|c|c|c|c|c|}
\hline & OR & $95 \%$ CI & $p$ value & $\mathrm{AOR}^{\uparrow}$ & $95 \% \mathrm{CI}$ & $p$ value \\
\hline \multicolumn{7}{|l|}{ Outcome } \\
\hline Probability of post-bereavement suicide attempt & 1.22 & $1.17-1.27$ & $<0.001$ & 1.19 & $1.14-1.25$ & $<0.001$ \\
\hline Adding perceived stigma of bereavement to above adjusted model & & & & 1.13 & $1.08-1.19$ & $<0.001$ \\
\hline Probability of post-bereavement suicide ideation & 1.26 & $1.22-1.30$ & $<0.001$ & 1.24 & $1.20-1.28$ & $<0.001$ \\
\hline Adding perceived stigma of bereavement to above adjusted model & & & & 1.18 & $1.13-1.22$ & $<0.001$ \\
\hline
\end{tabular}

${ }^{\uparrow}$ Adjusted for age, gender, socio-economic status, pre-bereavement depression, pre-bereavement (suicidal and non-suicidal) self-harm; and primary group size

\section{Sensitivity analysis}

In sensitivity analyses simulating potential biases introduced by $3 \%$ missing data for socio-economic status the magnitude, direction and precision of adjusted odds ratios for the association between loneliness scores and outcomes were unchanged.

\section{Discussion}

\section{Main findings}

The findings of this analysis of British cross-sectional data support our hypothesis that people who feel lonely after the sudden death of a friend or relative are more likely to experience suicidal thoughts and to make a suicide attempt after the loss, even when adjusting for social network size and for the perceived stigma of the bereavement. The magnitude of our risk estimates were such that the odds of making a suicide attempt or of having suicidal thoughts increased by $19 \%$ and $24 \%$, respectively with each point increase on our loneliness measure. Potential explanations for this association include loneliness contributing to substance misuse, to a sense that no-one would understand their problems, and to the intensity of fantasies about reunion with the deceased, otherwise considered a normal grief reaction. The associations remained significant after adding stigma to final models, and we found no interaction with high versus low stigma score. This is surprising because of the observed association between the stigma of sudden bereavement and suicidal ideation and attempt [22]. This suggests that a sense of loneliness is independently associated with probability of suicidality among people who experience sudden bereavement.

Our interaction tests found that feeling lonely had no greater effect on suicidality among people bereaved by suicide than in people bereaved by non-suicide deaths, despite findings from the same sample identifying the suicidebereaved as having a significantly higher probability of postbereavement suicide attempt [25]. This again is surprising, and suggests that for the suicide-bereaved other factors relating to suicide loss make additional contributions to their suicide risk. It is plausible that the stigma of suicide may condition the suicide-bereaved to normalise loneliness as an expected consequence of suicide loss. Instead, other factors influencing suicide risk are likely to include psychiatric 
disorder [18, 19], substance misuse [47], and a reluctance to seek help for mental health problems [48]. Risk of mood disorders and post-traumatic stress disorder are elevated in people bereaved by suicide [19]. People who have experienced suicide bereavement describe binge drinking or drug taking as a coping strategy in the immediate aftermath of the death [47]. They are also less likely to consult their general practitioner than bereaved controls, despite their greater probably of mental illness [19], which may be due to the stigma of suicide loss reducing motivation to seek help [49-53]. Selfstigma is certainly an important factor in dissuading people from seeking help for mental health problems, primarily due to reluctance to disclose a mental health condition [54]. Suicide bereavement is characterised not only by stigma, but also by a sense of responsibility, rejection, and shame when compared to bereaved controls [55]. A more detailed exploration of patterns of help-seeking for suicidality among the suicide-bereaved in relation to shame and stigma may be warranted, as it is possible that a reluctance to seek help accounts for their greater risk of suicide attempt. Finally, it is possible that carer burden prior to suicide loss might give rise to higher levels of pre-bereavement loneliness [56], although this requires specific testing.

In our sample there was a high overall proportion of people with post-bereavement suicidal ideation (47\%), greatly exceeding those for the maximum lifetime prevalence of suicidal ideation (20.6\%) in any corresponding age group within the APMS 2007 representative sample of the English population [36]. The proportion of those reporting a suicide attempt (7\%) in our sample, during a period spanning a mean of 5 years since their loss, was similar to the maximum lifetime prevalence of suicide attempt (7.3\%) in any corresponding age group within the same APMS 2007 representative sample [36]. Among a group in which suicidality is commonplace, screening for loneliness may be a useful means of identifying individuals at risk of suicide attempt and initiating a conversation about appropriate ways of alleviating loneliness in the context of that person's unique circumstances.

\section{Results in the context of other studies}

Few other studies have used nationwide population-based samples to investigate the associations of loneliness with suicide-related outcomes. Previous work in Canada found a strong association between loneliness and suicidal ideation (OR 10.5; 95\% CI 8.4-13.1) and self-harm (OR 13.5; 95\% CI 9.3-19.6) in a representative household sample [12]. However, that study measured loneliness using one unvalidated item capturing responses to the question "How often do you feel alone?", rated on a 5-point scale from 0 (never) to 4 (very often), and odds ratios were unadjusted [12]. An analysis of English household survey data found that those with higher levels of loneliness were significantly more likely to report past year suicidal ideation (AOR 11.09; 95\% CI 6.91-17.79) and past year suicide attempts (AOR 17.37; 95\% CI 5.51-54.72) [6]. This analysis adjusted for age, sex, educational qualifications, ethnicity, marital status, wealth, employment status, alcohol dependence, social support, physical health conditions, stressful life events and common mental disorder, and the magnitude of the effect sizes were large. However, confidence intervals were wide, and the analysis was based on the single item from the SFQ, whereas ours used the SFQ item together with seven complementary items capturing perceptions of belongingness, companionship, acceptance, and connectedness.

\section{Strengths and limitations}

We analysed data from a large, UK-wide sample of 3193 bereaved adults using a newly-validated loneliness measure with good face and content validity, and internal consistency, albeit without data validating it against an established measures of loneliness. Our hypotheses were formulated on the basis of current theory and clinical experience, and our models were adjusted for pre-selected potential confounders, including pre-bereavement psychopathology. Results were robust to sensitivity analysis simulating the biases potentially introduced by missing data. However, our use of cross-sectional data limits interpretation of the chronology of the pathways between loneliness scores (measured currently) and outcomes (measured at any time after the bereavement), and it is possible that suicidality engenders further loneliness or that the relationships are bidirectional. Our logistic regression did not capture the time between bereavement and suicidality outcome measures, nor whether respondents related their suicidality to the bereavement. As bereavement was as distal as 30 years for some participants, and a mean of 5 years, it is possible that the experience of current loneliness may relate to more recent factors, including current mental illness. It is also possible that suicidality since bereavement was explained by other unmeasured factors. Further longitudinal work is necessary to understand the directionality, and potential reinforcing effect, of these relationships, and how the strength of associations varies over time. Our sample was predominantly white, female and highly-educated, and this limits generalisability of our findings to settings outside HEIs and in other countries.

\section{Clinical and research implications}

This study has identified loneliness after a sudden bereavement to be associated with suicidality, whether or not the loss was due to suicide. Our findings suggest a need for 
agencies that support bereaved people, including counsellors, general practitioners, voluntary sector organisations, and informal networks, to inquire about loneliness and consider ways of addressing this. However, the stigma of admitting to feeling lonely may preclude identification of the problem, particularly in those who wish to mask their sense of isolation. As yet the evidence base for interventions that address loneliness and prevent adverse mental health outcomes is in its infancy. However, systematic reviews of studies evaluating the effectiveness of interventions to address loneliness in people with existing mental health problems [57] and in the general population [58]. These tentatively favour interventions addressing maladaptive cognitions, such as biases in cognition towards negative aspects of the social context. Pending trial evidence in bereaved populations, referral to a peer bereavement support group may be an acceptable means of increasing connectedness among bereaved people, particularly those who feel alienated from their non-bereaved peers. Such groups may provide the only access to people who can understand them, or the only setting in which expressing grief is acceptable [59]. They may also be a setting in which selective attention towards others' failure to offer support after loss, and other maladaptive cognitions, might be challenged. Even for those who have family and friends around them, peer support groups offer the potential for connectedness with people who have a shared understanding of the experience of bereavement, as well as the opportunity to help others [59].

Longitudinal work to explore the nature of the relationships between loneliness, stigma, social support, and suicidality, would help determine the comparative contribution of loneliness, stigma, and poor informal support as putative mediators of the association between sudden bereavement and suicide-related outcomes. Understanding these relationships would help determine whether there is a need to develop and trial individual-level or community-level anti-stigma interventions among those have been bereaved and who feel lonely and stigmatised. Investigating the relationship between loneliness and hopelessness is also warranted. Theoretical models of the interrelationship between loneliness and suicidal ideation suggest that the effects of loneliness on future suicidality operate through its influence on hopelessness [60]. However, when tested, findings support an alternative model in which hopelessness predicts both loneliness and suicidality, with no direct relationship between loneliness and suicidality beyond hopelessness [60]. Our study did not measure hopelessness, but testing these hypotheses in a bereaved population would contribute further to our understanding of these mechanisms. Finally, further investigation of the relationship between loneliness and entrapment is warranted using longitudinal approaches, as entrapment is a key factor in theoretical models of suicide [61].

\section{Conclusions}

Our results confirm an association between feeling lonely after the sudden death of a friend or relative and an increased risk of suicidal thoughts and suicide attempt, which is not explained by a lack of friends or family or by feeling stigmatised by the loss. Our most striking finding is that suicide bereavement is not associated with feeling lonely, and that loneliness had no greater effect in people bereaved by suicide, despite their greater risk of suicide attempt. Further work using longitudinal data is needed to understand the potentially complex associations between loneliness, stigma, mental illness, social support and suicidality in people who experience bereavement. This will help identify how we might mitigate the negative health effects of sudden bereavement, as part of suicide prevention efforts.

Acknowledgements This survey was funded by a Medical Research Council Population Health Scientist Fellowship Award to AP (G0802441). AP is currently supported by an ESRC (UKRI cross-council) grant to fund the Loneliness and Social Isolation in Mental Health research network (project reference: ES/S004440/1). AP, MK and DO are supported by the UCLH National Institute for Health Research (NIHR) Biomedical Research Centre (BRC). DO is also part supported by the NIHR Collaboration for Leadership in Applied Health Research and Care (CLAHRC) North Thames at Bart's Health NHS Trust. The funders had no role in design and conduct of the study; collection, management, analysis, and interpretation of the data; or preparation, review, and approval of the manuscript. The views expressed are those of the authors and not necessarily those of the funders. We would like to thank all the HEIs from England, Wales, Northern Ireland, and Scotland that consented to participate, listed below, and all the bereaved individuals who took time to respond to the online survey. Participating HEIs: Bishop Grosseteste University; Bournemouth University; Central School of Speech and Drama; City University; Cranfield University; Courtauld Institute; De Montfort University; University of Greenwich; King's College London; Liverpool Institute for Performing Arts; Liverpool John Moores University; London Metropolitan University; Norwich University of the Arts; Royal Veterinary College; School of Oriental and African Studies; St George's, University of London; Staffordshire University; Trinity Laban Conservatoire of Music and Dance; UCL; University of Suffolk; University of Bedfordshire; University of Chester; University of Cumbria; University of Leeds; University of Liverpool; University of Oxford; University of Southampton; University of Worcester; University of Westminster; Queen Margaret University; Heriot-Watt University; Scottish Agricultural College (now part of Scotland's Rural University College); University of Dundee; Cardiff University; Cardiff Metropolitan University (formerly University of Wales Institute Cardiff); Queen's University Belfast; University of Ulster.

\section{Compliance with ethical standards}

Conflict of interest The authors confirm that they have no conflicts of interest.

Open Access This article is licensed under a Creative Commons Attribution 4.0 International License, which permits use, sharing, adaptation, distribution and reproduction in any medium or format, as long as you give appropriate credit to the original author(s) and the source, 
provide a link to the Creative Commons licence, and indicate if changes were made. The images or other third party material in this article are included in the article's Creative Commons licence, unless indicated otherwise in a credit line to the material. If material is not included in the article's Creative Commons licence and your intended use is not permitted by statutory regulation or exceeds the permitted use, you will need to obtain permission directly from the copyright holder. To view a copy of this licence, visit http://creativecommons.org/licenses/by/4.0/.

\section{Appendix: Development of loneliness measure}

\section{Items}

\section{Loneliness item from the Social Functioning Questionnaire (SFQ)}

Participants provide fixed choice responses to the following statement, scored as:

"Almost all the time" $\square$ "Much of the time" $\square$ "Not usually" $\square$ "Not at all"

"I feel lonely and isolated from other people".

Measure of perceived social support, from the 1987 Health and Lifestyle survey in England:

Participants provide fixed choice responses to the seven statements below, scored as:

$\square$ not true $\square$ partly true $\square$ certainly true

"I would now like you to think about your family and friends. (By family I mean those who live with you as well as those elsewhere.) Here are some comments people have made about their family and their friends. For each statement, please say whether it is: not true, partly true, or certainly true for you".

- There are people I know amongst my family and friends who do things to make me happy.

- There are people I know amongst my family and friends who make me feel loved.

- There are people I know amongst my family and friends who can be relied on no matter what happens.

- There are people I know amongst my family and friends who would see that I am taken care of if I needed to be.

- There are people I know amongst my family and friends who accept me just as I am.

- There are people I know amongst my family and friends who make me feel an important part of their lives.

- There are people I know amongst my family and friends who give me support and encouragement.

In the UCL Bereavement Study these survey questions followed a set of questions on the social network, priming respondents to think about their wider social network, including friends, relatives, and acquaintances.

\section{Scoring}

The SFQ item is conventionally scored as: 0 ("Not at all"), 1 ("Not usually"), 2 ("Much of the time"), or 3 ("Almost all the time"), with higher scores indicating greater loneliness.

The measure of perceived social support was originally scored as: 1 ("not true"), 2 ("partly true") or 3 ("certainly true), generating total scores between 7 and 21, and transformed into three categories corresponding to no lack of perceived social support, a moderate lack, and a severe lack [38]. Other studies have scored items from 0 to 2 , generating total scores between 0 and 14, and demonstrating good internal consistency (Cronbach's alpha 0.88) [62]. For the current study we rescored these items in reverse order to $0,1,2$ to match the directionality of the SFQ item scoring, and reduced all scores by 1 to match the lowest scoring (0) for SFQ. This resulted in a measure scored from 0 to 17 , and with higher scores at the scale's negative pole (denoting a lack of belongingness, acceptance, connectedness and companionship).

\section{Tests of validity}

\section{Datasets}

To test construct validity of this eight-item measure we used two British cross-sectional datasets: the APMS general population samples for 2007 [36] and 2014 [63], and the UCL Bereavement Study sample [25]. The Department of Health's seven-yearly APMS national population surveys provide data on the prevalence of both treated and untreated psychiatric disorders in the English adult population aged 16 years and over. We included in our analysis of APMS data from 2007 and 2014 adults who had completed the single SFQ item on loneliness, and the seven items on perceived social support, and were aged below 65 years $(n=10,420)$. We included in our analysis of UCL Bereavement Study data those who had completed the same eight items $(n=3193)$. This sample included respondents who had experienced sudden bereavement due to sudden natural causes $(n=1952)$, sudden unnatural causes $(n=666)$, or suicide $(n=575)$.

\section{Construct validity}

We assessed construct validity by conducting Exploratory Factor Analysis (EFA) of the eight selected questionnaire items to ascertain which to retain. To do this we first used combined data on people aged below 65 in the APMS 2007 and 2014 household surveys. We used a more stringent eigenvalue threshold than the default value of 1 by the scree test (screeplot in Stata) to identify the natural bend or break 
point in the data curve, and used the number of data points above this break to identify the number of factors to retain [64]. We then derived a Kaiser-Meyer-Olkin (KMO) measure of sampling adequacy using the standard threshold of 0.7 . We summed all eight items and tested internal consistency of this scale by measuring Cronbach's alpha, and then retested this seven times, each time dropping one item to compare Cronbach's alpha values.

\section{Convergent validity}

To test convergent validity, a sub-type of construct validity, we tested univariate associations of the 8-item loneliness measure with measures theoretically related to the construct of loneliness. We chose variables capturing social connectedness, and tested for associations with the following using the nonparametric equality-of-medians test:

- marital status;

- primary group size, using a component of the Interview Measure of Social Relations (IMSR) as used in the 1987 Health and Lifestyle survey in England [38] (categorised as $0-3$ individuals/4 to $8 / 9$ or more);

- number of encounters with primary group members in the last week, using a different component of the IMSR, (categorised as: contact with no-one in last week; contact with 1-5 people; with 6-10 people; with $11-15$ people; with 16-20 people; with 21-30 people; with $31+$ people);

- 8 individual items within the Standardised Assessment of Personality-Abbreviated Scale (SAPAS) screen for personality disorders (difficulty with relationships, tendency to be a loner/mistrustful/to lose one's temper/impulsive/ anxious/dependent/perfectionist).

- screen for possible personality disorder, using the SAPAS [44] score with the standard cutoff at a threshold of four traits.

We also tested for an association with number of children, pre-hypothesising no association, in keeping with previous findings of no association between loneliness and the presence, number, and age of children for married men and women [65].

We then repeated these tests using the UCL Bereavement Study dataset. Our tests of convergent validity additionally tested for an association with a variable unavailable in the APMS dataset: living situation (categorised as: alone; living with spouse/partner; single parent living with children; living with parents; living with other relatives; sharing accommodation with non-relatives; student hall of residence or student hostel; temporary hostel or bed and breakfast accommodation; homeless; other).

\section{Factor analysis}

Our EFA of eight items using the APMS combined datasets showed that all items mapped to a one factor structure, with this factor having an eigenvalue of 3.87. In contrast, other factors' eigenvalues ranged from -0.15 to 0.18 . Factor loadings on the one factor identified were 0.32 for the SFQ item on perceived loneliness, and between 0.61 and 0.80 for the seven items on the reverse-scored perceived social support scale. KMO values on all items were above 0.89 (exceeding the standard threshold of 0.7 ), with an overall value of 0.91 . By convention, this confirmed that the variables had sufficient in common to warrant a factor analysis. On summing all eight items, Cronbach's alpha was 0.80 (designated by convention as strong internal consistency), with an average inter-item covariance of 0.08 . On retesting Cronbach's alpha by removing individual items, its value never dropped below 0.76, therefore denoting acceptable internal consistency and that all eight items should be retained. Our tests of convergent validity showed that the loneliness measure was significantly associated with all variables tested apart from number of children in the household.

Repeating these tests in the UCL Bereavement Study dataset, EFA again identified a one factor structure, with this factor having an eigenvalue of 3.59. On contrast, other factors' eigenvalues ranged from -0.15 to 0.12 . Factor loadings on the one factor identified were 0.44 for the SFQ item on perceived loneliness, and between 0.61 and 0.76 for the seven items on the reverse-scored perceived social support scale. KMO values on all items were above 0.89 (again exceeding the threshold), with an overall value of 0.91. On summing all eight items, Cronbach's alpha was 0.84 (again designating strong internal consistency), with an average inter-item covariance of 0.10 . On retesting Cronbach's alpha by removing individual items, its value again never dropped below 0.80 . Our tests of convergent validity showed that the loneliness measure was significantly associated with all variables tested apart from number of children in the household and perfectionistic traits, as pre-hypothesised. These findings established good face, content, construct, and convergent validity of this continuous measure.

\section{References}

1. WHO (2014) Preventing suicide: a global imperative. WHO, Geneva

2. De Jong Gierveld J (1998) A review of loneliness: concept and definitions, determinants and consequences. Rev Clin Gerontol 8:73-80

3. Fassberg MM, Van Orden KA, Duberstein P, Erlangsen A, Lapierre S, Bodner E, Canetto SS, De Leo D, Szanto K, Waern M 
(2012) A systematic review of social factors and suicidal behavior in older adulthood. Int J Environ Res Public Health 3:722-745

4. Wills TA (1991) Social support and interpersonal relationships. In: Clark MS (ed) Prosocial behavior: review of personality and social psychology. SAGE Publications Inc, New York, pp 265-289

5. Pitman A, Mann F, Johnson S (2018) Advancing our understanding of loneliness and mental health problems in young people. Lancet Psychiatry 5(12):955-956

6. Stickley A, Koyanagi A (2016) Loneliness, common mental disorders and suicidal behavior: findings from a general population survey. J Affect Disord 197(81):87

7. Koivumaa-Honkanen H, Honkanen R, Viinamaki H, Heikkilla K, Kaprio J, Koskenvuo M (2001) Life satisfaction and suicide: a 20-year follow-up study. Am J Psychiatry 158(3):433-439

8. Ma J, Batterham PJ, Calear AL, Sunderland M (2019) The development and validation of the Thwarted Belongingness Scale (TBS) for interpersonal suicide risk. J Psychopathol Behav Assess 41(3):456-469

9. O'Connor RC, Nock MK (2014) The psychology of suicidal behaviour. Lancet Psychiatry 1(1):73-85

10. Van Orden KA, Cukrowicz KC, Witte TK, Joiner TE (2012) Thwarted belongingness and perceived burdensomeness: construct validity and psychometric properties of the Interpersonal Needs Questionnaire. Psychol Assess 1:197-215

11. O'Connor RC, Kirtley OJ (2018) The integrated motivational volitional model of suicidal behaviour. Philos Trans R Soc Lond B Biol Sci 373:1754

12. Stravynski A, Boyer R (2011) Loneliness in relation to suicide ideation and parasuicide: a population-wide study. Suicide Life Threat Behav 31(1):32-40

13. Kuwert P, Knaevelsrud C, Pietrzak RH (2014) Loneliness among older veterans in the United States: results from the national health and resilience in veterans study. Am J Geriatr Psychiatry 22(6):564-569

14. Stein JY, Itzhaky L, Levi-Belz Y, Solomon Z (2017) Traumatization, loneliness, and suicidal ideation among former prisoners of war: a longitudinally assessed sequential mediation model. Front Psychiatry 8:281

15. Lasgaard M, Goossens L, Elklit A (2011) Loneliness, depressive symptomatology, and suicide ideation in adolescence: crosssectional and longitudinal analyses. J Abnorm Child Psychol 39(1):137-150

16. Westefeld JS, Maples MR, Buford B, Taylor S (2001) Gay, lesbian, and bisexual college students. J Coll Stud Psychother 15(3):71-82

17. Guldin MB, Kjaersgaard MIS, Fenger-Gron M, Parner ET, Li J, Prior A, Vestergaard M (2017) Risk of suicide, deliberate selfharm and psychiatric illness after the loss of a close relative: a nationwide cohort study. World Psychiatry 2:193-199

18. Pitman A, Osborn DPJ, King MB, Erlangsen A (2014) Effects of suicide bereavement on mental health and suicide risk. Lancet Psychiatry 1(1):86-94

19. Erlangsen A, Runeson B, Bolton JM, Wilcox HC, Forman JL, Krogh J, Shear MK, Nordentoft M, Conwell Y (2017) Association between spousal suicide and mental, physical, and social health outcomes: a longitudinal and nationwide register-based study. JAMA Psychiatry 74(5):456-464

20. Stroebe M, Stroebe W, Abakoumkin G (2005) The broken heart: suicidal ideation in bereavement. Am J Psychiatry 162(11):2178-2180

21. Pitman AL, Stevenson F, Osborn DPJ, King MB (2018) The stigma associated with bereavement by suicide and other sudden deaths: a qualitative interview study. Soc Sci Med 198:121-129

22. Pitman A, Rantell K, Marston L, King M, Osborn D (2017) Perceived stigma of sudden bereavement as a risk factor for suicidal thoughts and suicide attempt: analysis of British cross-sectional survey data on 3387 young bereaved adults. Int J Environ Res Public Health 14(3):286

23. Barrett TW, Scott TB (1989) Development of the grief experience questionnaire. Suicide Life Threat Behav 19(2):201-215

24. Pitman AL, Osborn DPJ, Rantell K, King MB (2016) The stigma perceived by people bereaved by suicide and other sudden deaths: a cross-sectional UK study of 3432 bereaved adults. J Psychosom Res 87:22-29

25. Pitman AL, Osborn DPJ, Rantell K, King MB (2016) Bereavement by suicide as a risk factor for suicide attempt: a cross-sectional national UK-wide study of 3432 young bereaved adults. BMJ Open 6(1):e009948

26. Bailley SE, Dunham K, Kral MJ (2000) Factor structure of The Grief Experience Questionnaire (GEQ). Death Stud 24(8):721-738

27. Keyes KM, Pratt C, Galea S, McLaughlin KA, Koenen KC, Shear MK (2014) The burden of loss: unexpected death of a loved one and psychiatric disorders across the life course in a national study. Am J Psychiatry 171(8):864-871

28. Piper WE, McCallum M, Joyce AS, Rosie JS, Ogrodniczuk JS (2001) Patient personality and time-limited group psychotherapy for complicated grief. Int J Group Psychother 51(4):525-552

29. Andriessen K, Draper B, Dudley M, Mitchell PB (2016) Pre- and postloss features of adolescent suicide bereavement: a systematic review. Death Stud 40(4):229-246

30. Scott HR, Pitman A, Kozhuharova P, Lloyd-Evans B (2020) A systematic review of studies describing the influence of informal social support on psychological wellbeing in people bereaved by sudden or violent causes of death. BMC Psychiatry 20:1

31. Pitman A, Krysinska K, Osborn D, King M (2012) Suicide in young men. Lancet 379(9834):2383-2392

32. Fortune S, Sinclair J, Hawton K (2008) Help-seeking before and after episodes of self-harm: a descriptive study in school pupils in England. BMC Public Health 8(1):1-13

33. Biddle L, Donovan J, Sharp D, Gunnell D (2007) Explaining non-help-seeking amongst young adults with mental distress: a dynamic interpretive model of illness behaviour. Sociol Health Illn 29(7):983-1002

34. McQueen C, Henwood K (2002) Young men in 'crisis': attending to the language of teenage boys' distress. Soc Sci Med 55(9):1493-1509

35. Pitman A, Osborn DPJ, King MB (2015) The use of internet-mediated cross-sectional studies in mental health research. BJPsych Adv 3:175-184

36. McManus S, Meltzer H, Brugha T, Bebbington P, Jenkins R (2009) Adult psychiatric morbidity in England, 2007. Results of a household survey. National Centre for Social Research, Leeds

37. Tyrer P, Nur U, Crawford M, Karlsen S, MacLean C, Rao B, Johnson $\mathrm{T}$ (2005) The social functioning questionnaire: a rapid and robust measure of perceived functioning. Int J Soc Psychiatry 51(3):265-275

38. Cox BD, Blaxter M, Buckle ALJ, Fenner NP, Golding J, Gore M, Huppert FA, Nickson J, Roth M, Stark J, Wadsworth MEJ, Wichelow M (1987) The health and lifestyle survey. Health Promotion Research Trust, London

39. Bebbington PE, Minot S, Cooper C, Dennis M, Meltzer H, Jenkins R, Brugha T (2010) Suicidal ideation, self-harm and attempted suicide: results from the British psychiatric morbidity survey 2000. Eur Psychiatry 25(7):427-431

40. Spiers N, Bebbington PE, Dennis MS, Brugha TS, McManus S, Jenkins R, Meltzer H (2014) Trends in suicidal ideation in England: the National Psychiatric Morbidity Surveys of 2000 and 2007. Psychol Med 44(01):175-183

41. ONS (Office for National Statistics), "Standard Occupational Classification 2010 Volume 3 The National Statistics Socio-economic 
classification: (Rebased on the SOC2010) User Manual. HMSO ," HMSO,2010.

42. Robins LN, Wing JK, Wittchen H-U, Helzer JE, Babor T, Burke J, Framer AE, Jablensky A, Pickens R, Regier D (1988) The Composite International Diagnostic Interview. An epidemiologic instrument for use in conjunction with different diagnostic systems and in different cultures. Arch Gen Psychiatry 45:1069-1077

43. Moran P, Leese M, Lee T, Walters P, Thornicroft G, Mann A (2003) Standardised Assessment of Personality-Abbreviated Scale (SAPAS): preliminary validation of a brief screen for personality disorder. Br J Psychiatry 183(3):228-232

44. Germans S, Van Heck GL, Moran P, Hodiamont PPG (2008) The Self-report Standardized Assessment of Personality-abbreviated Scale: preliminary results of a brief screening test for personality disorders. Personality 2(2):70-76

45. Brugha T, Sturt E, MacCarthy B, Potter J, Wykes T, Bebbington P (1987) The Interview Measure of Social Relationships: the description and evaluation of a survey instrument for assessing personal social resources. Soc Psychiatry 22(2):123-128

46. Brugha T, Weich S, Singleton N, Lewis G, Bebbington P, Jenkins R, Meltzer H (2005) Primary group size, social support, gender and future mental health status in a prospective study of people living in private households throughout Great Britain. Psychol Med 35(5):705-714

47. Eng J, Drabwell L, Stevenson F, King M, Osborn D, Pitman A (2019) Use of alcohol and unprescribed drugs after suicide bereavement: qualitative study. Int J Environ Res Public Health 16(21):4093

48. Pitman A (2018) Addressing suicide risk in partners and relatives bereaved by suicide. Br J Psychiatry 4:197-198

49. Cvinar JG (2005) Do suicide survivors suffer social stigma: a review of the literature. Perspect Psychiatr Care 41(1):14-21

50. Hawton K, Simkin S (2003) Helping people bereaved by suicide. BMJ 327(7408):177-178

51. Knieper AJ (1999) The suicide survivor's grief and recovery. Suicide Life Threat Behav 29(4):353-364

52. Provini C, Everett JR, Pfeffer CR (2000) Adults mourning suicide: self-reported concerns about bereavement, needs for assistance, and help-seeking behaviour. Death Stud 24(1):1-19

53. Walter T (1999) On bereavement: the culture of grief. Open University Press, Buckingham

54. Clement S, Schauman O, Graham T, Maggioni F, Evans-Lacko S, Bezborodovs N, Morgan C, Rusch N, Brown JSL, Thornicroft
$\mathrm{G}$ (2014) What is the impact of mental health-related stigma on help-seeking? A systematic review of quantitative and qualitative studies. Psychol Med 45:1-17

55. Kolves K, Zhao Q, Ross V, Hawgood J, Spence SH, De Leo D (2019) Suicide and other sudden death bereavement of immediate family members: an analysis of grief reactions 6-months after death. J Affect Disord 243:96-102

56. Van Baarsen B, Van Duijn MAJ, Smit JH, Snijders TAB, Knipscheer KPM (2002) Patterns of adjustment to partner loss in old age: the widowhood adaptation longitudinal study. Omega (Westport) 44(1):5-36

57. Mann F, Bone JK, Lloyd-Evans B, Frerichs J, Pinfold V, Ma R, Wang J, Johnson S (2017) A life less lonely: the state of the art in interventions to reduce loneliness in people with mental health problems. Soc Psychiatry Psychiatr Epidemiol 52(6):627-638

58. Masi CM, Chen HY, Hawkley LC, Cacioppo JT (2011) A metaanalysis of interventions to reduce loneliness. Pers Soc Psychol Rev 3:219-266

59. Tal Young I, Iglewicz A, Glorioso D, Lanouette N, Seay K, Ilapakurti M, Zisook S (2012) Suicide bereavement and complicated grief. Dialogues Clin Neurosci 14(2):177-186

60. Joiner TE, Rudd MD (1996) Disentangling the interrelations between hopelessness, loneliness, and suicidal ideation. Suicide Life Threat Behav 26(1):19-26

61. Forkmann T, Teismann T (2017) Entrapment, perceived burdensomeness and thwarted belongingness as predictors of suicide ideation. Psychiatry Res 357:84-86

62. Wickham S, Taylor P, Shevlin M, Bentall RP (2014) The impact of social deprivation on paranoia, hallucinations, mania and depression: the role of discrimination social support, stress and trust. PLoS ONE 9(8):e105140

63. McManus S, Bebbington P, Jenkins R, Brugha T (2016) Mental health and wellbeing in England. Adult Psychiatric Morbidity Survey 2014. NHS Digital, Leeds

64. Costello A, Osborne J (2005) Best practices in exploratory factor analysis: four recommendations for getting the most from your analysis. Pract Assess Res Eval 10:7

65. Gove WR, Geerken MR (1977) The effect of children and employment on the mental health of married men and women. Soc Forces 56(1):66-76 